\title{
Preliminary Clinical Trial of Balance Compensation System for Improvement of Balance in Patients With Spinocerebellar Ataxia
}

\author{
Ji Seon Hong, $\mathrm{MD}^{1}$, Ji Hyun Kim, MD, $\mathrm{PhD}^{1}$, Sang Yeol Yong, $\mathrm{MD}^{1,2}$, Young Hee Lee, MD, PhD ${ }^{1,2}$, \\ Sung Hoon Kim, MD, PhD ${ }^{1,2}$, Jun Young Park, $\mathrm{MD}^{1}$, Jung Kuk Lee ${ }^{3}$, Ji Yoon Jang, MD \\ ${ }^{1}$ Department of Rehabilitation Medicine, Wonju Severance Christian Hospital, Yonsei University Wonju College of Medicine, Wonju; \\ ${ }^{2}$ Yonsei Institute of Sports Science and Exercise Medicine, Wonju; \\ ${ }^{3}$ Department of Biostatistics, Yonsei University Wonju College of Medicine, Wonju, Korea
}

\begin{abstract}
Objective To determine the immediate and short-term impact of the application of wearable balance compensation system (BCS) on balance impairment in patients with spinocerebellar ataxia (SCA).

Methods The study enrolled 6 participants with SCA with varying degrees of balance impairment. After adjustment for individual fitting, wearable BCS with up to $3 \%$ body weight was placed in a garment on the trunk. Sway direction and magnitude were measured with sensors placed posteriorly at the lumbosacral junction, immediately before and after, and at day 1 , day 2, and day 7 after wearing the BCS. Timed Up \& Go test (TUG) and 25 -foot timed walk test were performed, and static foot pressure was measured.

Results A significant improvement in static and dynamic balance was found during the 25 -foot timed walk and in static foot pressure measurement results after wearing the BCS, when compared with that at baseline ( $\mathrm{p}=0.044 \mathrm{vs}$. $\mathrm{p}=0.011$ ). Anterior and posterior sway showed improvements from baseline after wearing the BCS. Improvement in the lateral swaying movement control was also seen.

Conclusion Application of the BCS might be beneficial in the improvement ofthe static and dynamic balance in patients with SCA. Further research on long-term effects and with a larger sample size is indicated.
\end{abstract}

Keywords Balance impairment, Spinocerebellar ataxia, Rehabilitation

Received September 30, 2019; Revised October 25, 2019; Accepted October 28, 2019; Published online August 5, 2020 Corresponding author: Ji Yoon Jang

Department of Rehabilitation Medicine, Wonju Severance Christian Hospital, Yonsei University Wonju College of Medicine, 20 Ilsan-ro, Wonju 26426, Korea. Tel: +82-33-741-1455, Fax: +82-33-741-1455, E-mail: raichu11@naver.com

ORCID: Ji Seon Hong (http://orcid.org/0000-0002-7412-2145); Ji Hyun Kim (http://orcid.org/0000-0001-9061-3350); Sang Yeol Yong (http://orcid. org/0000-0003-0288-4121); Young Hee Lee (http://orcid.org/0000-0001-6861-4250); Sung Hoon Kim (http://orcid.org/0000-0001-6043-7640); Jun Young Park (http://orcid.org/0000-0001-5721-4112); Jung Kuk Lee (http://orcid.org/0000-0003-1874-449X); Ji Yoon Jang (http://orcid.org/0000-00027635-8901).

@ This is an open-access article distributed under the terms of the Creative Commons Attribution Non-Commercial License (http://creativecommons.org/ licenses/by-nc/4.0) which permits unrestricted noncommercial use, distribution, and reproduction in any medium, provided the original work is properly cited. Copyright $\odot 2020$ by Korean Academy of Rehabilitation Medicine 


\section{INTRODUCTION}

Spinocerebellar ataxia (SCA) is a progressive, autosomal dominant neurodegenerative disorder that affects the cerebellum and its connected structures, resulting in the impairment of balance and ataxia [1]. Patients with cerebellar ataxia along with postural disorders often experience frequent falls. Fonteyn et al. [2] reported that $84 \%$ of the patients with cerebellar ataxia fell at least once, during a 1-year study conducted among 113 patients with degenerative ataxia. The traumatic consequences of falls increase with the number of incidents. Fear of falling must also be screened for because it can accelerate the loss of personal independence; thus, the prevention of falls is important [3]. The cerebellum plays an important role in motor learning (including postural learning), and the involvement of the cerebellum in the disease might therefore limit the effectiveness of rehabilitation programs in the treatment of balance disorders of cerebellar origin $[4,5]$. Patients with SCA have difficulty in maintain the balance due to the variability of lesions affecting multiple neurologic processes. Patients with SCA have deficits in somatosensory, visual, and vestibular functions. SCA also affects central processing and activation of the motor output; however, due to the variability in the location of the lesions and their severity, there are no typical gait patterns or balance dysfunction identified with SCA. The presence of ataxia can further complicate the clinical picture. One-third of the patients with multiple sclerosis (MS) along with cerebellar atrophy present with cerebellar ataxia, which is a significant cause of balance and gait problems [6]. Despite the frequency and significance of balance impairments in this population, rehabilitative solutions remain challenging. Clinicians have used several strategies including aerobic exercise [7], resistance training [8-11], balance and gait retraining [12], and neurodevelopmental techniques $[13,14]$ to address the balance and gait dysfunction in patients with MS.

One upcoming intervention for MS patients with ataxia involves adding weights to the torso or extremities to assist in coordinated movements for the function orgait [14-19]. Lucy and Hayes [19] showed that weight training of the shoulder reduced the lateral sway in 10 patients with ataxia (3 had MS). Balanced-body-torso-weight training (BBTW) showed immediate advantages over a non-weight training therapy in gait velocity and over a standardized weighted condition, for functional activity in people with MS who are ambulatory but have balance and mobility abnormalities [20,21].

Other than BBTW, balance compensation system (BCS) is another method of adjusting the weight distribution in a weight vest. The difference between BCS and BBTW is that, in the BBTW system, additional weight placed on the torso is calculated and adjusted manually. In contrast, in BCS, a premade software program calculates the subject's dynamic body balance pattern and decides the location of additional weights.

To the best of our knowledge, no study has investigated the effectiveness of a wearable BCS vest in patients with SCA. To study the effects of weight training more specifically in people with SCA, we investigated whether adding an individualized small amount of weight to the trunk of patients with SCA improves their balance and function. The hypothesis of this research was that use of the BCS would improve the measures of gait and balance impairments in patients with SCA.

\section{MATERIALS AND METHODS}

\section{Subjects}

Participants were recruited through the Yonsei University Wonju Christian Hospital. The inclusion criteria were patients (1) who could walk at least 35 feet with or without a cane or a walker and (2) who could stand at least 10 seconds without support. Participants were excluded if they had conditions that would limit their ability to undergo and tolerate the test and treatment, or had complete blindness or current back pain. Such conditions were identified through a medical screening questionnaire administered at baseline. All subjects provided informed consent, and this study was approved by the Institutional Review Board of Yonsei University Wonju Christian Hospital (No. CR318145).

\section{Experimental design}

Subjects served as their own control in this single group, non-blinded study. Each subject performed the first set of both conditions: at baseline without wearing a BCS vest, and wearing a BCS vest for 18 hours except when sleeping during the 7-day intervention. 


\section{Procedures}

Using motion-sensors (MTw Awinda wireless 3DOF motion tracker + dongle by Xsens) equipped with a gyrosensor and an accelerometer, we measured the imbalance pattern in 6 subjects with neurodegenerative disorder causing motor-impaired test subjects when the subjects were asked to walk in a pre-determined path. More specifically, the subjects were asked to walk in "the ambulation measurement path" two times and changes in the pitch, roll, and yaw angles of the subjects' torso were measured. The measurement data were analyzed using Propriologics software (AnchorLogics, New York, NY, USA) to determine the dynamic balance pattern of the subjects.

When the dynamic balance pattern of the subject was outside of the pre-stored "normal value" (i.e., the dynamic balance pattern value of the people without balance problems), Propriologics software provided weight placement suggestions indicating the size and locations of the weights to be placed on the subject's torso to correct the balance of the subject. In our experiment, the dynamic balance pattern values of all 6 subjects were outside the normal value.

Using the output obtained from Propriologics, a weighted vest was made to be worn by each subject. The weighted vest used in this study was made of polyester material on the outside and Velcro loop in the inside, and the weights from Flex-Metal Weight products (Ironwear, LaPorte, TX, USA), which can be attached and detached to and from the vest (Fig. 1), were used. The weights used in this study were in the units of $1 / 8 \mathrm{lbs}(56.7 \mathrm{~g}), 1 / 4 \mathrm{lbs}$ (113.4 g), and 1/2 lbs (226.8 g). For optimal results, the total weight used on test subjects was approximately $3 / 100$ of the body weight of each subject.

After a weight-loaded vest was customized for the subject, the subject walked on the ambulation measurement path, this time, wearing the weight-loaded vest and the motion-sensors. The measurement data were analyzed using Propriologics software to determine whether the dynamic balance pattern value of the subject was corrected. The weight placement adjustment process was repeated until an optimal result was obtained (i.e., the subject's dynamic balance pattern value was closer to the normal value).

For initial screening, the subjects completed a questionnaire consisting of detailed questions about comor-
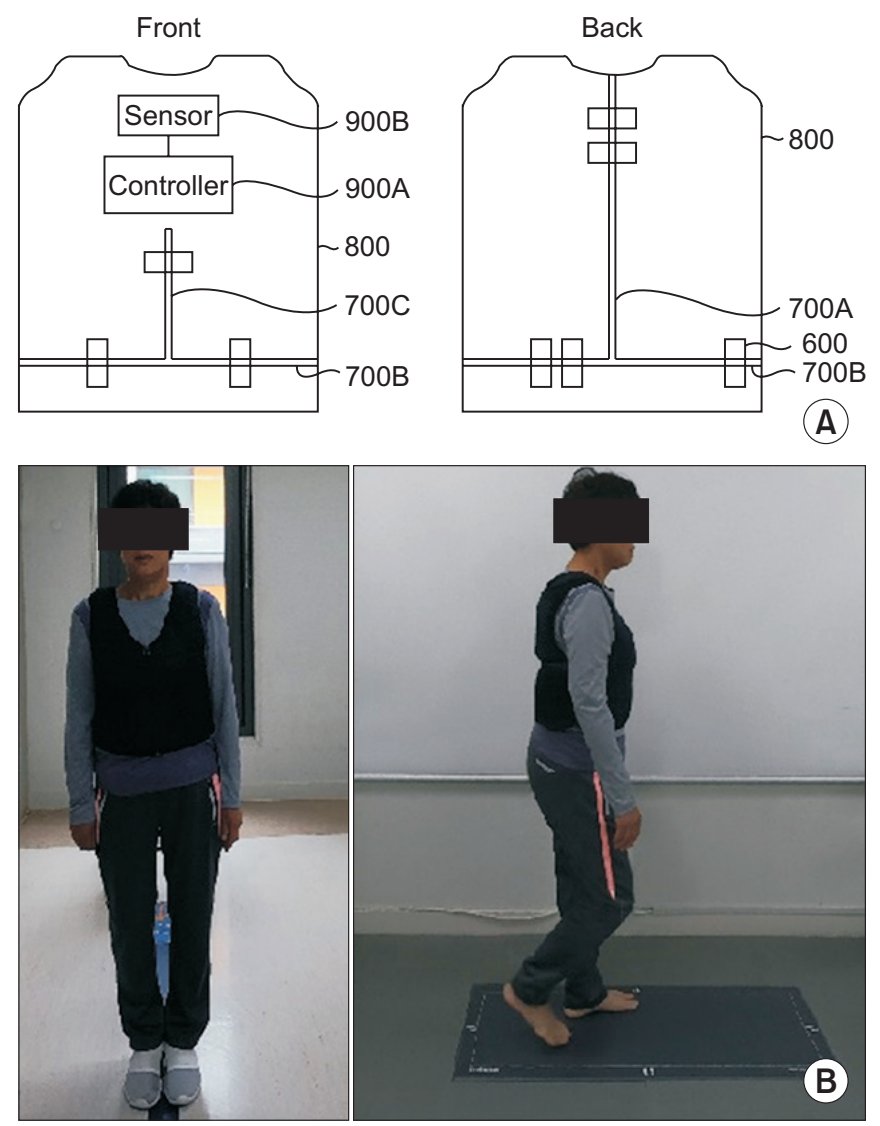

Fig. 1. (A) The schematic appearance of weighted BCS vest. It was made of polyester material on the outside and Velcro loop in the inside, and the weights, which can be attached and detached to and from the vest. Wireless motion-sensors areequipped with a gyrosensor and an accelerometer, connected with controller. (B) Patients are performing 25-foot walk test and static foot scan test to evaluate static and dynamic balance. BCS, balance compensation system.

bidities, balance, falls, injuries, sensory changes, age, and weight. Fortesting at each period (baseline, and days 1,2 , and 7 with BCS vest), the subjects performed a set of 4 tasks in the following order: computerized sway test, Timed Up \& Go test (TUG), a timed 25-foot walk, and static foot pressure measurement using the footscan system (RSscan International, Paal, Belgium). These tests are validated and commonly used for evaluating balance and ambulatory performance, as they representthe static (computerized sway test, static foot pressure measurement) and dynamic (TUG and 25-foot walk) balance. Throughout the testing, the subjects were guarded against falls. The TUG was timed from the point subjects 
rose from a chair, walked $3 \mathrm{~m}$ to a line on the floor, turned around, walked back to the chair, until they sat down with their back against the back of the chair. The subjects were allowed 1 practice trial before the actual test. For the 25 -foot walk test, the subjects were instructed to walk as quickly as they comfortably could, to a line about 3 feet beyond the 25 -foot mark. Timing was initiated when the subject started walking at the 0 -foot line and ended when one foot crossed the 25 -foot line. Static foot pressure was measured as the subjects were guided to stand on the footscan system for 15 seconds to assess the trajectory of the center of pressure (COP) in the static position. Analysis of the COP trajectory was done using a custom-made software (Footscan version 9.5.10). The assessments of the degree of $\mathrm{COP}$ alteration for static balance evaluation were done by calculating the total ellipse area of the COP trajectories.

All data were analyzed using SPSS version 25.0 (IBM, Armonk, NY, USA) and SAS version 9.4 (SAS Institute Inc., Cary, NC, USA). Dependent variables were analyzed based on the data from the different time periods (baseline, days 1, 2, and 7 with wearable BCS vest) using repeated measures of Kruskal-Wallis test and a single regression test for the TUG, 25-foot walk, and static balance measurement scores to analyze statistical significance. Dunn procedure was used for multiple comparisons with compensated significance level by Bonferroni method. A p-value less than 0.05 was considered to indicate statistical significance.

\section{RESULTS}

Seven participants between the age of 12 and 67 years were recruited. Six subjects met the inclusion and exclusion criteria. Five subjects completed all the tests (base-

Table 1. Subject characteristics

\begin{tabular}{cccc}
\hline Subject & Age (yr)/Sex & Months since Dx & Falls $^{\text {a) }}$ \\
\hline 1 & $41 / \mathrm{M}$ & 37 & 0 \\
2 & $51 / \mathrm{F}$ & 16 & $>1$ \\
3 & $33 / \mathrm{M}$ & 80 & 1 \\
4 & $12 / \mathrm{M}$ & 44 & $>1$ \\
5 & $62 / \mathrm{F}$ & 13 & $>1$ \\
6 & $67 / \mathrm{M}$ & 35 & 1 \\
\hline
\end{tabular}

Dx, diagnosis.

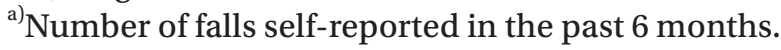

line, days 1, 2, and 7 days with the BCS vest) and one subject completed the tests only at baseline and day 7 after wearing the BCS vest. All data obtained from this subject were included. A summary of the subjects' characteristics is shown in Table 1.

Two (33.3\%) of the 6 subjects were women. The mean age of the subjects was 44.2 years and the mean time since SCA diagnosis was 37.5 months. Five subjects (83.3\%) had fallen at least once in the past 6 months. A fall was defined as losing balance and unexpectedly landing on the floor. During the walk tests, 2 subjects used a cane. During TUG and 25-foot timed walk test, the test was not repeated because of patient fatigue.

\section{Static balance of computerized sway analysis and COP measurement}

The static balance measurement scores are shown in Table 2. Regression analysis of the COP trajectory ellipse area from the baseline condition to the condition after wearing the BCS vest revealeda significant decrease $(\mathrm{p}=0.011)$ (Fig. 2). Kruskal-Wallis analysis showed a significant correlation in the improvement of static balance (decrease in ellipse area) between baseline and day 7 after wearing the BCS vest $(\mathrm{p}=0.003)$.

\section{Dynamic balance of incorporating gait}

The TUG and 25-foot walk test scores are shown in Table 3. The TUG results demonstrated decreased test performance time between baseline and after the BCS application, but no significant differences were found across the different time periods $(\mathrm{p}=0.065)$. However, there was a negative correlation between TUG and time ( $\beta=-1.667)$ (Fig. 3). Repeated measures of regression analysis for the 25 -foot walk scores showed significant correlation in the

Table 2. Static balance measurement of COP trajectory ellipse area

\begin{tabular}{ccccc}
\hline \multirow{2}{*}{ Subject } & \multicolumn{4}{c}{ COP trajectory ellipse area $\left(\mathbf{m m}^{\mathbf{2}}\right)$} \\
\cline { 2 - 5 } & Baseline & Day $\mathbf{1}$ & Day $\mathbf{2}$ & Day $\mathbf{7}$ \\
\hline 1 & 48 & 25 & 4 & 10 \\
2 & 198 & 49 & 18 & 10 \\
3 & 24 & 13 & 5 & 19 \\
4 & 240 & 42 & 114 & 44 \\
5 & 33 & 56 & 30 & 62 \\
6 & 49 & - & - & 8 \\
\hline
\end{tabular}

$\mathrm{COP}$, center of pressure. 
duration to complete the test performance $(\mathrm{p}=0.044)$ (Fig. 4). Kruskal-Wallis analysis showed no significant correlation between the TUG and 25-foot walk scores across the time periods. As there was not adequate number of participants to compare the statistical correlation, the analysis showed a positive correlation in improved dynamic balance across time. This finding indicates that adequate number of participants might show a significant correlation.

\section{DISCUSSION}

In this study, we investigated the immediate and shortterm effects of wearing a weight-loaded BCS vest in 6 participants with SCA. The results showed positive effects of

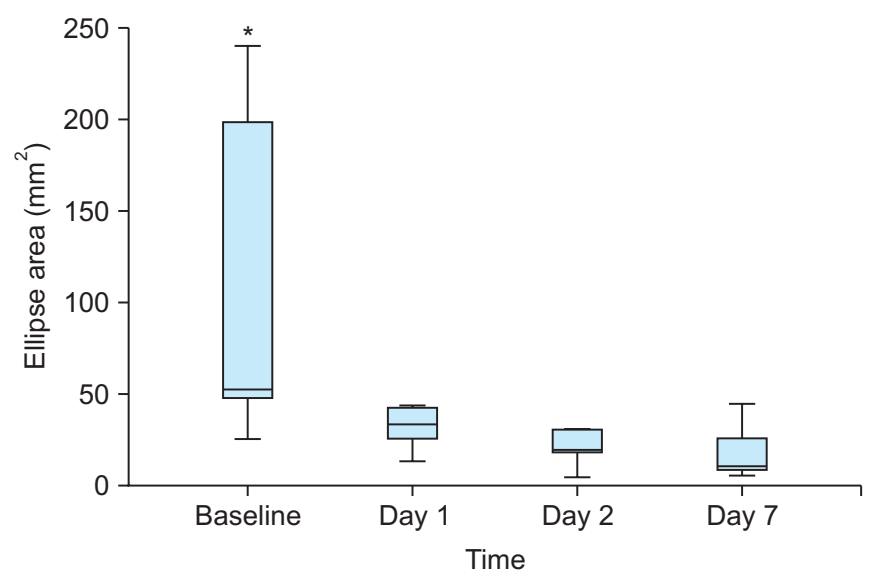

Fig. 2. Box-plot of mean difference in total ellipse area of COP trajectory results from baseline to day 7. Mean ellipse area showed substantial decrease day 1 after application of BCS vest. Mean difference showed statistically significant decrease across time $(\mathrm{p}=0.011)$. COP, center of pressure; BCS, balance compensation system. ${ }^{*} \mathrm{p}<0.05$.
BCS in improving the static and dynamic balance in SCA patients.

Static balance measurements results showed immediate as well as short-term effects (after 7 days of wearing the BCS vest) in the improvement of static balance. The trajectory of COP movement showed a rapid reduction in the ellipse area after applying BCS in the standing posture. Similar effects were shown by Lucy and Hayes [19]; they reported that these positive effects might occur due to improved lateral balance but might be less effective in improving the anterior-posterior swaying. Hunt et al. [22] reported an improvement in the COP displacement after BBTW application in MS patients. A research by Crittendon et al. [20] also reported that application of weights improves control in the lateral swaying movement but does not improve the anterior-posterior swaying move-

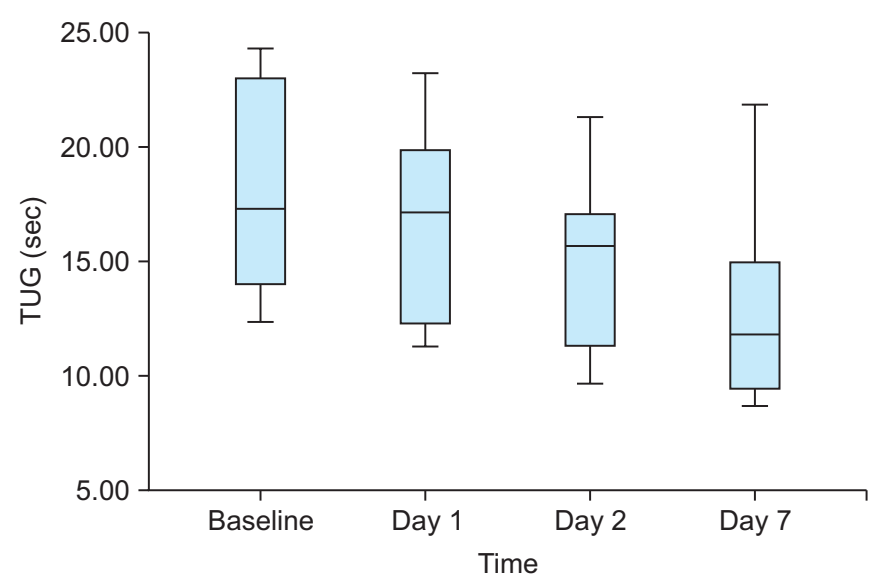

Fig. 3. Box-plot of mean difference in TUG results from baseline to day 7. Mean difference of TUG results had no statistically significant correlation $(\mathrm{p}=0.065)$. However, there were negative correlation between TUG and time ( $\beta=-1.667)$. TUG, Time Up \& Go test.

Table 3. Dynamic balance test of TUG and 25-foot walk test

\begin{tabular}{|c|c|c|c|c|c|c|c|c|}
\hline \multirow{2}{*}{ Subject } & \multicolumn{4}{|c|}{ TUG (sec) } & \multicolumn{4}{|c|}{ 25-foot walk test (sec) } \\
\hline & Baseline & Day 1 & Day 2 & Day 7 & Baseline & Day 1 & Day 2 & Day 7 \\
\hline 1 & 12.35 & 11.24 & 9.65 & 8.69 & 8.02 & 7.04 & 5.99 & 4.64 \\
\hline 2 & 23.05 & 19.89 & 15.73 & 13.33 & 14.06 & 8.26 & 8.56 & 7.72 \\
\hline 3 & 15.48 & 12.31 & 11.35 & 10.29 & 15.78 & 6.91 & 6.08 & 5.66 \\
\hline 4 & 19.23 & 17.18 & 17.06 & 15.00 & 16.35 & 16.02 & 15.32 & 13.16 \\
\hline 5 & 24.35 & 23.26 & 21.35 & 21.90 & 16.53 & 16.82 & 13.08 & 12.13 \\
\hline 6 & 14.05 & - & - & 9.42 & 8.16 & - & - & 6.92 \\
\hline Mean \pm SD & $18.085 \pm 4.49$ & $16.776 \pm 4.52$ & $15.028 \pm 4.17$ & $13.105 \pm 4.51$ & $13.123 \pm 3.70$ & $11.01 \pm 4.45$ & $9.806 \pm 3.78$ & $8.372 \pm 3.68$ \\
\hline
\end{tabular}

TUG, Time Up \& Go test. 


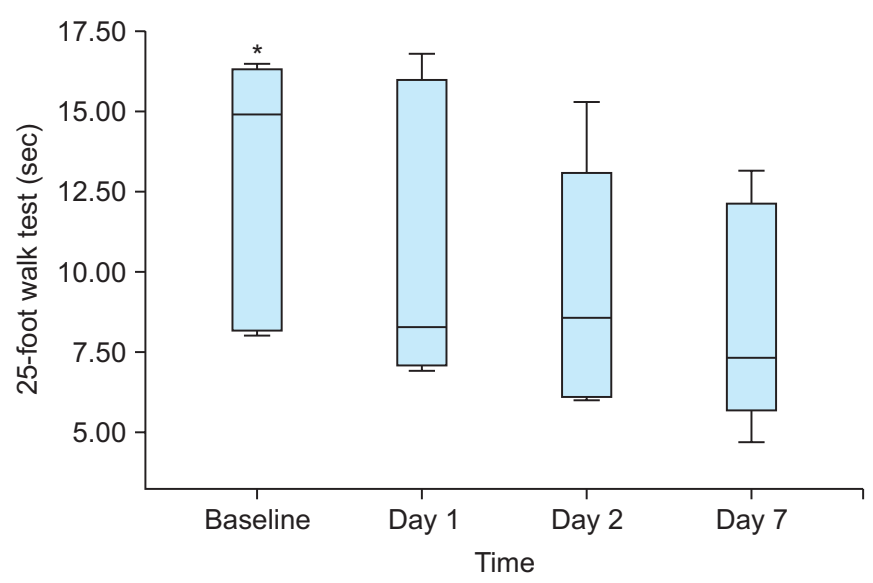

Fig. 4. Box-plot of mean difference in 25-foot timed walk test trial results from baseline to day 7 . Mean difference showed statistically significant decrease in performance time from baseline to day 7 after BCS application $(\mathrm{p}=0.044)$. BCS, balance compensation system. ${ }^{*} \mathrm{p}<0.05$.

ment in MS patients. In their study, the direction of maximum weight placement did not match the direction of change in the average COP. However, participants in this study showed a decrease in COP trajectory ellipse area as well as a more central COP. Data showed decreased movement of $\mathrm{COP}$ along the $\mathrm{X}$ - and $\mathrm{Y}$-axis in the standing posture across time periods. These findings suggest that BCS can improve not only the lateral balance but also the anterior-posterior balance. The difference between the results of our study and those of previous studies might due to the difference in the methods for adjusting the weight distribution, as the BCS uses a computerized analysis, while BBTW uses a manual analysis technique.

Improvement in the TUG test scores demonstrates an increase in the functional ability (sit to stand, turns and gait) and dynamic balance compared with the baseline status. While it showed no significance in the regression analysis, a qualitative difference such as a decrease in the time to complete the test was observed. Participants showed more fluent movements during walking, turning, and sit to stand exercise. These findings suggest that more quantifiable results can be detected using motion analysis and electromyography in future research.

The 25 -foot walk test can assess gait velocity in dynamic gait balance. In our study, it showed a significant increase in gait velocity across time with regression analysis. Crittendon et al. [20] reported that adding weights increased the gait velocity compared with gait without weight addi- tion, which is similar to the results derived from our previous study. Other features influencing gait pattern, such as step length, duration of stance, and swing phase was not evaluated in this study. Further research with gait analysis to evaluate and quantify the effects of BCS and to explore whether it can improve gait pattern or increase the step length might be beneficial.

The precise mechanism for improvements in the static and dynamic balance with weight loading is not yet fully understood. Previous studies reported that weight loading can not only improve balance and gait ataxia, but also motor control and intention tremor $[15,16,23]$. Widener et al. [24] reported several hypotheses for the mechanism of immediate therapeutic effects in weight-bearing vests. One possible mechanism in postural improvement is that weight loading changes the body's center of gravity [24]. Previous studies reported that participants resisted a greater rotational force for a longer time with weight loading than when without weights [20]. These findings suggest that weight loading induces participants to resist weight, producing a counter force to the weight force, leading to improvement in the regulation of trunk balance.

Another proposed alternative mechanism is the apparent integration of sensory stimuli provided by these light weights and the consequent adjustment of postural sway, improvements in stability with perturbations and rotational forces, and increases in gait velocity [25]. Immediate improvements with BBTW include joint compression and increased afferent input from the body segments (joint capsules, articular fat pads, and intra- and extracapsular ligaments). Additional sensory inputs facilitate and improve motor responses and also increase the conscious awareness, leads to long-term effects $[18,25]$. However, Morgan and his colleagues $[16,17]$ reported that effectiveness of weights on ataxic limbs does not seem to diminish over time. These long-term effects might be due to relearning effects of the body, improving response to somatosensory stimuli and proprioception. Our research showed persistent therapeutic effects in the short-term (7 days) during BCS application. Additional trials for longterm effects should be investigated in the future.

Previous studies mainly focused on patients with MS alongwith ataxia. In our study, the results showed that weight-loaded vests can be a method to improve ataxia in patients with cerebellar atrophy, known as an intractable 
disease. While BCS is an unfamiliar system for adjusting weighted vests, the results and data showed improvement in static and dynamic balance in addition to immediate and short-term therapeutic effects.

Our study has several limitations. First, there was a relatively small number of participants in this study. Second, the patients were not tested without weight loading cross match condition during the follow-up tests at days 1,2 , and 7 after first application of BCS vest. Third, patients were followed up until 7 days after the application, which is a relatively short period of time to evaluate the longterm effects. Another limitation is that patients were not evaluated in randomized control trials with non-vested or simple vested conditions, which can minimize the learning effect in the patients and affect the results of 25foot walk test and TUG test. Future research should compare the effectiveness of BCS with BBTW as well as longterm effects of BCS in a larger population with balance impairment and ataxia.

In summary, improvements in balance were noted when weight-loaded BCS vest was applied to patients with SCA. The results of our study suggest that BCS could have a positive therapeutic effect for improving balance in patients with lower extremity ataxia. Future research will be necessary to confirm the effectiveness of BCS compared with simple weight loading vest condition using randomized controlled trials and long-term followup trials to evaluate the delayed effects of BCS in patients with SCA or other diseases involving balance problems.

\section{CONFLICT OF INTEREST}

No potential conflict of interest relevant to this article was reported.

\section{AUTHOR CONTRIBUTION}

Conceptualization: Hong JS, Jang JY. Methodology: Hong JS, Kim SH, Jang JY. Formal analysis: Hong JS, Yong SY, Lee JK, Jang JY. Project administration: Hong JS, Yong SY, Jang JY. Visualization: Hong JS, Kim JH, Park JY. Writing - original draft: Hong JS, Jang JY. Writing - review and editing: Hong JS, Kim JH, Kim SH, Lee YH, Jang JY. Approval of final manuscript: all authors.

\section{REFERENCES}

1. Marquer A, Barbieri G, Perennou D. The assessment and treatment of postural disorders in cerebellar ataxia: a systematic review. Ann Phys Rehabil Med 2014; 57:67-78.

2. Fonteyn EM, Schmitz-Hubsch T, Verstappen CC, Baliko L, Bloem BR, Boesch S, et al. Prospective analysis of falls in dominant ataxias. Eur Neurol 2013;69:537.

3. Gaxatte C, Nguyen T, Chourabi F, Salleron J, Pardessus V, Delabriere I, et al. Fear of falling as seen in the multidisciplinary falls consultation. Ann Phys Rehabil Med 2011;54:248-58.

4. Ioffe ME, Chernikova LA, Ustinova KI. Role of cerebellum in learning postural tasks. Cerebellum 2007;6:8794.

5. Manto M. The cerebellum, cerebellar disorders, and cerebellar research: two centuries of discoveries. Cerebellum 2008;7:505-16.

6. Weinshenker BG, Issa M, Baskerville J. Long-term and short-term outcome of multiple sclerosis: a 3-year follow-up study. Arch Neurol 1996;53:353-8.

7. Rodgers MM, Mulcare JA, King DL, Mathews T, Gupta SC, Glaser RM. Gait characteristics of individuals with multiple sclerosis before and after a 6-month aerobic training program. J Rehabil Res Dev 1999;36:183-8.

8. Taylor NF, Dodd KJ, Prasad D, Denisenko S. Progressive resistance exercise for people with multiple sclerosis. Disabil Rehabil 2006;28:1119-26.

9. Gutierrez GM, Chow JW, Tillman MD, McCoy SC, Castellano V, White LJ. Resistance training improves gait kinematics in persons with multiple sclerosis. Arch Phys Med Rehabil 2005;86:1824-9.

10. White LJ, McCoy SC, Castellano V, Gutierrez G, Stevens JE, Walter GA, et al. Resistance training improves strength and functional capacity in persons with multiple sclerosis. Mult Scler 2004;10:668-74.

11. Wiles CM, Newcombe RG, Fuller KJ, Shaw S, FurnivalDoran J, Pickersgill TP, et al. Controlled randomised crossover trial of the effects of physiotherapy on mobility in chronic multiple sclerosis. J Neurol Neurosurg Psychiatry 2001;70:174-9.

12. Lord SE, Wade DT, Halligan PW. A comparison of two physiotherapy treatment approaches to improve walking in multiple sclerosis: a pilot randomized con- 
trolled study. Clin Rehabil 1998;12:477-86.

13. Smedal T, Lygren H, Myhr KM, Moe-Nilssen R, Gjelsvik $B$, Gjelsvik $O$, et al. Balance and gait improved in patients with MS after physiotherapy based on the Bobath concept. Physiother Res Int 2006;11:104-16.

14. Chase RA, Cullen JK Jr, Sullivan SA, Ommaya AK. Modification of intention tremor in man. Nature 1965; 206:485-7.

15. Hewer RL, Cooper R, Morgan MH. An investigation into the value of treating intention tremor by weighting the affected limb. Brain 1972;95:579-90.

16. Morgan MH, Hewer RL, Cooper R. Application of an objective method of assessing intention tremor: a further study on the use of weights to reduce intention tremor. J Neurol Neurosurg Psychiatry 1975;38:259-64.

17. Morgan MH. Ataxia and weights. Physiotherapy 1975; 61:332-4.

18. Clopton N, Schultz D, Boren C, Porter J, Brillbart T. Effects of axial weight loading on gait for subjects with cerebellar ataxia: preliminary findings. Neurol Rep 2003;27:15-21.

19. Lucy SD, Hayes KC. Postural sway profiles: normal subjects and subjects with cerebellar ataxia. Physio- ther Can 1985;37:140-8.

20. Crittendon A, O'Neill D, Widener GL, Allen DD. Standing data disproves biomechanical mechanism for balance-based torso-weighting. Arch Phys Med Rehabil 2014;95:43-9.

21. Widener GL, Allen DD, Gibson-Horn C. Randomized clinical trial of balance-based torso weighting for improving upright mobility in people with multiple sclerosis. Neurorehabil Neural Repair 2009;23:784-91.

22. Hunt CM, Widener G, Allen DD. Variability in postural control with and without balance-based torsoweighting in people with multiple sclerosis and healthy controls. Phys Ther 2014;94:1489-98.

23. Holmes G. The cerebellum of man. Brain 1939;62:130.

24. Widener GL, Allen DD, Gibson-Horn C. Balancebased torso-weighting may enhance balance in persons with multiple sclerosis: preliminary evidence. Arch Phys Med Rehabil 2009;90:602-9.

25. Goff B. The application of recent advances in neurophysiology to Miss M. Rood's concept of neuromuscular facilitation. Physiotherapy 1972;58:409-15. 